\title{
Differentiation of Paired Human Subcutaneous and Visceral Adipose Tissues by Holistic Proteome Profiling using LC-MS/MS: a Pilot Study
}

\author{
Paul MM van Haard ${ }^{* 1}$, Paul Herbrink², Dave H Schweitzer ${ }^{3}$ \\ ${ }^{1 *}$ Dept. of Clinical Chemistry, Medical Laboratories, Reinier de GraafGasthuis, Delft, The \\ Netherlands \\ ${ }^{2}$ Dept. of Medical Immunology, Medical Laboratories, Reinier de GraafGasthuis, Delft, The \\ Netherlands \\ ${ }^{3}$ Dept. of Internal Medicine and Endocrinology, Reinier de GraafGasthuis, Delft, The Netherlands \\ haard@rdgg.nl,p.herbrink@casema.nl,D.Schweitzer@rdgg.nl
}

\begin{abstract}
:
Background: We examined, for the first time proteins expressions and abundancies within paired human subcutaneous (SAT) and visceral adipose tissues (VAT), using equal amounts of fully digested proteins.

Methods: Fully digested protein preparations from white adipose tissues biopsies of an obese male patient were analyzed both directly and after prior fractionation using LC-MS/MS. Data were processed to yield identified proteins and protein abundances. Proteins were annotated to the three Gene Ontology (GO) domains: Cellular Components (CC), Biological Processes (BP) and Molecular Functions (MF).

Results: Without prior fractionation, 519 proteins were identified in SAT and 567 in VAT. The tissues had 458 proteins in common, accounting for $90 \%$ and $83 \%$ of identified proteins in SAT and VAT, respectively. With prior fractionation, 1307 proteins were identified in SAT and 1710 in VAT. The tissues had 1248 proteins in common, accounting for $95 \%$ and $73 \%$ of identified proteins in SAT and VAT, respectively. Semi-quantitative proteins abundances were compared for proteins occurring in common, unveiling large differences between the two tissues. Substantial differences were found in GO annotated proteins abundances, unique to either SAT or VAT, concerning extracellular, nucleus, cytoplasm, and membrane (CC), metabolic process and cellular component movement (BP), metal ion binding, nucleotide binding, RNA binding, protein binding and DNA binding $(M F)$.
\end{abstract}

Conclusion: The proteomes of paired human adipose tissues reflect both common and unique proteins and semi-quantitative proteins abundances. Our holistic analysis accounts as an alternative approach to look for biological differences between SAT and VAT in white adipose tissues.

Keywords: Liquid chromatography, mass spectrometry, adipose tissues, human, visceral, subcutaneous, proteomics.

\section{INTRODUCTION}

Metabolic syndrome and obesity are an increasing world-wide problem. One of the treatment modalities for the metabolic syndrome is weight reduction. When successful, diets lead to a rapid improvement in blood sugar levels and blood pressure. When normal-weight people gain modest amounts of fat, they do so by enlarging adipocytes in their upper body subcutaneous fat depot (SAT). By contrast, gain of lower body fat is largely the result of increasing the number of adipocytes, which persist when people lose weight again. Metabolic syndrome and obesity are associated with an increased risk of type 2 diabetes mellitus and cardiovascular disease.

In humans, white adipose tissue is located under the skin (subcutaneous adipose tissue; SAT) and around internal organs and in the liver (visceral adipose tissue; VAT). Apart from adipocytes (cells for fat storage, insulation and bioactive compounds production) the stromal vascular cells fraction (SVF) is present, including preadipocytes, mesenchymal stem cells, fibroblasts, endothelial cells, endothelial progenitor cell, T cells, B cells, adipose tissue macrophages and immune cells, such as neutrophils, eosinophils, lymphocytes, natural killer cells, and mast cells. Adipose tissue contains 
many small blood vessels. To date, adipose tissue derived hormones include adiponectin, resistin, plasminogen activator inhibitor-1 (PAI-1), TNF $\alpha$, IL-6, leptin, monocyte chemo attractant protein-1, and estradiol (E2). Adipose tissues also secrete adipocytokines (cell-to-cell signalling proteins), playing a role in obesity-associated complications (for Review: 1). In non-obese subjects there is a balance between so-called pro- and anti-inflammatory adipocytokines. In obese subjects this balance is evidently distorted due to both increased production of proinflammatory adipocytokines and decreased production of anti-inflammatory adipocytokines (for Review: 2). There is also evidence for a greater impact of both visceral adipose tissue (VAT) and central obesity on the inflammatory process (which contributes to manifest cardiovascular disease), rather than total body adipose tissue. To date, visceral obesity is associated with a state of chronic, low-grade inflammation, which may cause obesity-induced insulin resistance and a state of hypercoagulability (for Review: 3 ).

While the genome of tissues, and thus of adipose tissue may be considered static, the expression of that genome as proteins in a specific tissue is constantly changing due to the influence of environmental and physiological conditions. In adipose tissues, therefore intra-individual variances of proteins expressions estimated by time series measurements are expected to be very large; moreover, in living humans they can not be studied, neither ethically nor practically.

Measuring differences in the proteins expressions between types of adipose tissues from the same human may provide important information on common or unique processes and functions, at a specific timepoint. Also, monitoring changes in proteins expressions in response to stressors may provide important information on underlying processes. This may lead to a better understanding of both organ and disease processes in humans (4-5).

Adipose tissue proteomics is the exploration of the adipose tissue proteome: the entire complement of proteins expressed by adipose tissue genome under a specific set of conditions at a specific time. The adipose tissue secretome (i.e. the entire set of secretable proteins) is of interest when examining adipose tissue secretory dysfunctioning. LC-MS based proteomics enables the exploration of differential proteins expressions in tissues, cells and body fluids (6).

Different mass spectrometers can be used, and a number of separation and fractionation steps on either proteins or proteolytic peptides (digested proteins) can be performed to reduce sample complexity before applying LC-MS in 1 or more dimensions. Depending on sample characteristics the most ideal techniques have to be applied.

In this pilot study, gradient LC was used in combination with MS/MS analysis to assess the holistic proteomes of SAT and VAT, injecting native or prior offline fractionated proteolytic peptides. This choice of pre-analytical methods does influence the subset of proteins that will be identified. Prior to LC-MS/MS analysis, tissue samples may be processed separately into plasma membrane, cytosolic, mitochondrial or lipid droplet fractions. Adipose tissues contain many different populations of cells, each of which may play an unique role in adipose tissue functioning. Kheterpal et al. (7) profiled the proteome of stromal vascular cells and of mature adipocytes from human subcutaneous adipose tissue using a quantitative 2D gel LC-MS/MS method. Holistic proteome mapping by LC-MS/MS of paired human adipose tissues has not been performed before. Four adipose deposits, containing two types of adipose tissues have been mapped in goats recently (8). Their holistic method, using fractionation encountered less interference by high abundant proteins present in SAT and VAT. Global analyses of proteins present in the human adipose tissue have been performed by Hess and Chen (9), using nonholistic LC-MS/MS methodologies.

The first step in the present study on paired human adipose tissues proteomics is protein identification and profiling in a well-characterized holistic set-up, to provide a representative view of proteins present. We focus more on the experimental setup, the analytical methods and interpretation of outcome data rather than on the biology.

The analysis of proteins in paired adipose tissues is important for a better understanding of biological processes in adipose tissues of genders, in metabolic syndrome and obesity. Moreover, the goal of this pilot study is to compare the protein profiles of human visceral (omentental) adipose tissue (VAT) and subcutaneous (peripheral) adipose tissue (SAT) in an obese male patient, who underwent elective gallbladder surgery. The described combination of techniques is the most suitable for our research goals to achieve a sensitive identification of the complex sets of proteins, i.e. proteomes in human adiposity. 


\section{Materials AND Methods}

\subsection{Ethics}

This pilot study is part of an approved comparative study of the effect of caloric restriction on (adipo-) cytokine production of adipose tissues. Approval for this study was obtained from the reviewing, accredited Ethics Committee at Leiden, The Netherlands. All patients had to review, understand, agree to, and personally sign and date the Informed Consent form prior to enrolment in the study.

\subsection{Subject}

Samples of VAT and SAT were obtained from an overweighed (BMI 29.4), male patient, 31 years of age. Written informed consent was obtained from the patient prior to enrolment in the study.

\subsection{Tissue Sampling}

During gallbladder surgery, biopsies were taken from the omentum (visceral adipose tissue, VAT) and from subcutaneous (peripheral) adipose tissue (SAT). Biopsy samples were marked, placed in plastic tubes and sent directly to the medical laboratories in Delft. Biopsy material was aliquoted and was immediately stored frozen at $-80^{\circ} \mathrm{C}$. Samples were transported on dry ice $\left(-78.5^{\circ} \mathrm{C}\right)$ to the Radboud Proteomics Center in Nijmegen, The Netherlands.

\subsection{Sample Preparation for LC-MS/MS Analysis}

Essentially, proteins were extracted and digested essentially according to methods described by Wessels et al. (10). Briefly, proteins were extracted by mechanical grinding in $8 \mathrm{M}$ Urea/10 mMTris$\mathrm{HCl} \mathrm{pH=8}$ using a sample grinding kit (GE Healthcare). Samples were not heated above $37^{\circ} \mathrm{C}$, during the whole digestion procedure. In our experience, using this procedure protein modification due to reaction with urea is neglegible. Protein concentration was determined using the 2D Quant kit (GE Healthcare). An amount of $40 \mu \mathrm{g}$ total protein of each adipose tissue sample (in $8 \mathrm{M}$ Urea/10 mMTris- $\mathrm{HCl} \mathrm{pH}=8$ ) was diluted $1: 1$ with $50 \mathrm{mM}$ ammonium bicarbonate and subsequently reduced and alkylated for 30 minutes at room temperature by addition of $20 \mathrm{nmoldithiotreitol} \mathrm{and} 100 \mathrm{nmol} 2$ chloroacetamide, respectively. Proteins were then first digested with LysC for 3 hours at room temperature using a 1:50 (w/w) LysC:protein ratio. Next, the sample was diluted 1:3 (v/v) with 50 $\mathrm{mM}$ ammonium bicarbonate for overnight tryptic digestion at $37^{\circ} \mathrm{C}$ using a 1:50 (w/w) trypsin:protein ratio. After digestion, each sample was loaded on a STAGE-tip (Stop and Go Elution, C18-reversed phase SPE) for desalting and concentrating according to Rappsilber, Ishihama and Mann (11).

\subsection{Offline Peptide Fractionation}

In order to fractionate the protein digests for more in-depth proteome analysis using a 2 dimensional LC approach, we used an Acquity ultra-high performance liquid chromatography system (Waters Chromatography) coupled to a 1260 FC-AS Fraction collector (Agilent Technologies); the peptide mixtures were separated at $\mathrm{pH}=10$ into 20 fractions. We injected the digest (equivalent to $40 \mu \mathrm{g}$ total protein) on a Waters X-Bridge C18 column $(3.5 \mu \mathrm{m}$ particle size, $130 \AA$ pore size, $1 \mathrm{~mm}$ x $100 \mathrm{~mm}$, Waters Chromatography). Peptides were separated at a flow rate of $50 \mu 1 / \mathrm{min}$ using a linear gradient of 5-35\% acetonitrile with $20 \mathrm{~m}$ Mammoniumformate $\mathrm{pH}=10$ as mobile phase A and acetonitrile as mobile phase B (for details see Supplementary information). In total, 20 fractions of 1 minute were collected for each sample. Each fraction was evaporated to dryness using a SpeedVac (Eppendorf) and taken up in $10 \mu 10.1 \%$ formic acid for subsequent LC-MS/MS analysis with LC separation at $\mathrm{pH}=2.7$.

\subsection{LC-MS/MS Analysis}

Unfractionated samples and fractions from the offline LC separation were analyzed by LC-MS/MS on a nano Advance LC system coupled to an ultra-high resolution quadrupole time-of-flight mass spectrometer (UHR-QTOF MS) (MaXis 4G ETD, Bruker Daltonics). For unfractionated samples the equivalent of $1 \mu \mathrm{g}$ protein digest was injected and for fractionated samples $50 \%$ of each fraction was injected per LC-MS/MS analysis.

LC separations were performed using a trap setup and separating peptides on a reversed phase C18 column at $\mathrm{pH}=2.7$ using a linear acetonitrile gradient from 5\%-35\% containing $0.1 \%$ formic acid (see Supporting Information for details). For unfractionated samples, a 120 minutes gradient was used and 
for fractions from the offline LC fractionation 60 minutes gradients were used in the $2^{\text {nd }}$ dimension LC. The nanoflow LC system was coupled on line to the UHR-QTOF MS via a Captive Sprayer ionization source (Bruker Daltonics). Data dependent MS/MS spectra were obtained using collision induced dissociation (CID) as fragmentation technique. Relevant liquid chromatography and detailed mass spectrometry settings are given in the Supporting Information.

\subsection{Mass Spectrometry Data Analysis}

Data analysis was performed with the Max Quant software (Version 1.5.0.0; http://www. maxquant. org/; for reference see: 12), in combination with the NCBI RefSeq55 Homo Sapiens database. We used the NCBI database since it is a well annotated, curated and redundant protein database. An alternative would be the Uniprot Human database. Parameters used in data analysis by Max Quant are given in detail in the Supporting Information. iBAQ (output of Max Quant) in combination with a spike-in of a protein standard mixture with known molar concentration, converts peptide intensities measured by mass spectrometry into absolute protein amounts. In this pilot study, we could not make use of a protein standard mixture with known molar concentration, but we reasoned that the iBAQ algorithm (with at least 3 peptides per protein and no replicate analyses of a sample) could still be used to for comparison of intensities of identical proteins between adipose tissues. An alternative approach NSAF (see http://research.stowers.org/proteomics/Quant.html) was not possible; NSAF takes into account the sample-to-sample variation that is obtained when carrying out replicate analyses of a sample and the fact that longer proteins tend to have more peptide identifications than shorter proteins."

Protein annotations according to Gene Ontology (GO-Project; for reference see: 12) were acquired using Protein Center software (Version 3.12.10015; Thermo Scientific).

\subsection{Descriptive Statistics}

Gene Ontology domains annotations (13) were analyzed using Stat Graphics Centurion software (Version 16.2.04 for MS Windows; Statpoint, Inc., VA, USA). The comma separated terms in the standard GO tables cells were split using an MS-Excel formula and the resulting separate columns were transposed to one long-format column, thereby repeating their native GO domain terms. Columns indicating the presence of each protein named by their FASTA header (14) in SAT and/or VAT were conserved. Descriptive statistics for both domains and domains terms were thus obtained. IBAQ intensities and GO domains terms were coupled through FASTA header ("protein name") using an MS Access database. The coupled output contained protein name, GO domain and GO domain term and iBAQ intensity; the latter was converted afterwards to Log10-iBAQ-Intensity and this numerical information versus iBAQ Intensity rank order per common protein interpreted graphically using scatterplots as used by Perdomo et al. (15).

\section{RESUlts}

\subsection{Protein Identification in Two Types of Human Adipose Tissues}

For adipose tissue, we adopted a general proteomics workflow that combines the advantages of extensive proteome solubilization with the benefits of in-solution digestion (10). In an effort to generate a proteomic atlas that accurately captures the proteome, we performed 2 pilot experiments with LC-MS/MS measurements on proteome extracts from SAT and VAT samples.

A total number of 519 and 567 individual proteins were found in SAT and VAT, respectively without prior fractionation of the proteolytic peptides. The paired adipose tissues had 458 proteins in common, amounting to $90 \%$ and $83 \%$ of the total number of proteins in SAT and VAT, respectively. Therefore, 61 proteins were unique to SAT and 109 proteins to the paired VAT. An overview of identified proteins during the pilot study experiment 1 in SAT and VAT, shared in common or unique to either SAT or VAT is given in the Supporting Information.

Fractionation lowers the complexity of the proteolytic peptides mixture to be analyzed by LC-MS/MS (8). Although the total amount of analysis time spent on a biological sample is increased by fractionation, more MS/MS spectra can be recorded for lower abundant peptides that would be missed without fractionation. With samples other than adipose tissues the executive laboratory (Radboud Proteomics Center, Nijmegen, The Netherlands) experienced to find up to 2 times more proteins using prior fractionation (2D LC-MS/MS) using the 20 minutes gradients (this study), numbers comparable 
Differentiation of Paired Human Subcutaneous and Visceral Adipose Tissues by Holistic Proteome Profiling using LC-MS/MS: a Pilot Study

with numbers found with SDS-PAGE fractionation. As a result of our fair choice, a total number of 1307 proteins (5433 peptides) and 1710 proteins (8235 peptides) were identified in SAT and VAT, respectively. With prior offline fractionation, the paired adipose tissues had 1248 proteins in common, amounting to $95 \%$ and $73 \%$ of the total number of proteins in SAT and VAT, respectively. Therefore, 59 proteins were unique to SAT and 462 proteins to the paired VAT. An overview of proteins, identified by applying 2D LC-MS/MS in SAT and VAT, shared in common or unique to either SAT or VAT after fractionation is also given in the Supporting Information.

\subsection{Semi-Quantitative Information on Protein Concentration}

Comparison of MS/MS Intensities of identified proteins (using their iBAQ Intensity ratios in tissues) is only feasible for common proteins in paired SAT and VAT. Without fractionation, the MS/MS iBAQ Intensities Ratios VAT/SAT showed an interval [ $<0.01-56.2]$ with mean 1.8 and median 1.0 and a very skewed distribution. With fractionation, the MS/MS iBAQ Intensities Ratios VAT/SAT showed an interval [0.01-786.3] with mean 4.4 and median 2.1 and a very skewed distribution (Supporting Information). Proteins with either highest or lowest intensities turned out not to be identical in the two stages of our pilot experiments. We made no further use of data from the unfractionated LC-MS/MS experiment 1.

\subsection{Distribution of Identified Proteins amongst the Three GO Domains and Domains Terms}

Origins of proteins and proteins distributions in SAT and VAT in our male patient amongst the three standardized Gene Ontology domains Cellular Components (CC), Biological Processes (BP) and Molecular Functions (MF) are presented in Table 1 (all proteins annotations), Table 2 (common proteins annotations) and Table 3 (unique proteins annotations).

Table1. Statistical descriptive of all proteins found by LC-MS/MS in paired human adipose tissues, annotated to the three Gene Ontology domains and the domains terms

\begin{tabular}{|l|l|l|l|l|}
\hline & \multicolumn{2}{|c|}{ SAT } & \multicolumn{2}{l|}{ VAT } \\
\hline GO Domain & No. & Frequency \% & No. & Frequency \% \\
\hline Cellular Components & & & & \\
\hline Golgi & 100 & 2.6 & 130 & 2.7 \\
\hline Cell Surface & 79 & 2.0 & 86 & 1.8 \\
\hline Chromosome & 40 & 1.0 & 53 & 1.1 \\
\hline Cytoplasm & 729 & 18.9 & 933 & 19.1 \\
\hline Cytoskeleton & 215 & 5.6 & 247 & 5.1 \\
\hline Cytosol & 548 & 14.2 & 699 & 14.3 \\
\hline Endoplasmic reticulum & 118 & 13.1 & 157 & 3.2 \\
\hline Endosome & 61 & 1.6 & 79 & 1.6 \\
\hline Extracellular & 190 & 4.9 & 212 & 4.3 \\
\hline Membrane & 730 & 18.9 & 920 & 18.8 \\
\hline Mitochondrion & 235 & 6.1 & 3.1 & 6.2 \\
\hline Nucleus & 521 & 13.5 & 695 & 14.2 \\
\hline Organelle lumen & 164 & 4.2 & 189 & 309 \\
\hline Proteasome & 35 & 0.9 & 39 & 0.8 \\
\hline Ribosome & 40 & 1.0 & 56 & 1.2 \\
\hline Splicesomal Complex & 28 & 0.7 & 42 & 0.9 \\
\hline Vacuole & 34 & 0.9 & 47 & 1.0 \\
\hline Total & $\mathbf{3 8 6 7}$ & $\mathbf{1 0 0}$ & $\mathbf{4 8 8 5}$ & $\mathbf{1 0 0}$ \\
\hline
\end{tabular}

Table2. Statistical descriptive of common proteins found by LC-MS/MS in paired human adipose tisssues, annotated to the thee Gene Ontology domains and the domains terms

\begin{tabular}{|l|l|l|}
\hline GO Domain & No & Frequency (\%) \\
\hline Cellular Components & & \\
\hline Golgi & 96 & 2.6 \\
\hline Cell Surface & 77 & 2.1 \\
\hline Chromosome & 38 & 1.0 \\
\hline Cytoplasm & 705 & 19.0 \\
\hline Cytoskeleton & 207 & 5.6 \\
\hline Cytosol & 527 & 14.2 \\
\hline Endoplasmic reticulum & 114 & 3.1 \\
\hline Endosome & 60 & 1.6 \\
\hline
\end{tabular}


Paul MM van Haard et al.

\begin{tabular}{|l|l|l|}
\hline \hline Extracellular & 177 & 4.8 \\
\hline Membrane & 697 & 18.7 \\
\hline Mitochondrion & 229 & 6.2 \\
\hline Nucleus & 504 & 13.6 \\
\hline Organelle lumen & 157 & 4.2 \\
\hline Proteasome & 34 & 0.9 \\
\hline Ribosome & 39 & 1.1 \\
\hline Splicesomal Complex & 25 & 0.7 \\
\hline Vacuole & 33 & 0.9 \\
\hline Total & $\mathbf{3 7 1 9}$ & $\mathbf{1 0 0}$ \\
\hline
\end{tabular}

Table3. Statistical descriptive of unique proteins found by LC-MS/MS in paired human adipose tissues, annotated to the three Gene Ontology domains and the domains terms

\begin{tabular}{|c|c|c|c|c|}
\hline & SAT & VAT & & \\
\hline GO Domain & No. & Frequency \% & No. & Frequency \% \\
\hline \multicolumn{5}{|l|}{ Cellular Components } \\
\hline Golgi & 4 & 2.7 & 34 & 2.9 \\
\hline Cell Surface & 2 & 1.4 & 9 & 0.8 \\
\hline Chromosome & 2 & 1.4 & 15 & 1.3 \\
\hline Cytoplasm & 24 & 16.2 & 228 & 19.6 \\
\hline Cytoskeleton & 8 & 5.4 & 40 & 3.4 \\
\hline Cytosol & 21 & 14.2 & 172 & 14.8 \\
\hline Endoplasmic reticulum & 4 & 2.7 & 43 & 3.7 \\
\hline Endosome & 1 & 0.7 & 19 & 1.6 \\
\hline Extracellular & 13 & 8.8 & 35 & 3.0 \\
\hline Membrane & 33 & 22.3 & 223 & 19.1 \\
\hline Mitochondrion & 6 & 4.1 & 72 & 6.2 \\
\hline Nucleus & 17 & 11.5 & 191 & 16.4 \\
\hline Organelle lumen & 7 & 4.7 & 32 & 2.7 \\
\hline Proteasome & 1 & 0.7 & 5 & 0.4 \\
\hline Ribosome & 1 & 0.7 & 17 & 1.5 \\
\hline Splicesomal Complex & 3 & 2.0 & 17 & 1.5 \\
\hline Vacuole & 1 & 0.7 & 14 & 1.2 \\
\hline Total & 148 & 100 & 1166 & 100 \\
\hline \multicolumn{5}{|l|}{ Biological Processes } \\
\hline Cell Communication & 3 & 1.8 & 18 & 1.4 \\
\hline Cell death & 7 & 4.1 & 41 & 3.1 \\
\hline Cell differentiation & 5 & 2.9 & 35 & 2.7 \\
\hline Cell division & 0 & 0.0 & 10 & 0.8 \\
\hline Cell growth & 1 & 0.6 & 5 & 0.4 \\
\hline $\begin{array}{l}\text { Cell organization and } \\
\text { biogenesis }\end{array}$ & 19 & 11.2 & 159 & 12.0 \\
\hline Cell proliferation & 1 & 0.6 & 24 & 1.8 \\
\hline $\begin{array}{l}\text { Cellular component } \\
\text { movement }\end{array}$ & 9 & 5.3 & 33 & 2.5 \\
\hline Cellular homeostasis & 2 & 1.2 & 16 & 1.2 \\
\hline Coagulation & 5 & 2.9 & 14 & 1.1 \\
\hline Defense response & 7 & 4.1 & 45 & 3.4 \\
\hline Development & 1 & 0.6 & 11 & 0.8 \\
\hline Metabolic process & 38 & 22.4 & 336 & 25.4 \\
\hline $\begin{array}{l}\text { Regulation of biological } \\
\text { process }\end{array}$ & 32 & 18.8 & 257 & 19.4 \\
\hline Reproduction & 0 & 0.0 & 2 & 0.2 \\
\hline Response to stimulus & 26 & 15.3 & 188 & 14.2 \\
\hline
\end{tabular}

In this obese, male patient the most abundantly found numbers of proteins (frequency ranked) belong to the GO terms "cytoplasm", "membrane", "cytosol", "nucleus" in the domain CC, "metabolic process", "regulation of biological process", "response to stimulus", "cell organization and biogenesis" in the domain BP, and "protein binding", "catalytic activity", "nucleotide binding" in the domain MF (arbitrarily chosen abundant frequencies > 10\%; Table 1 and Table 2) in both types of adipose tissues. 
Differences between the overall most abundant frequencies -for all proteins- between SAT and VAT range only 0.1-1.0 PPts (percentage points; signed), but still may concern decades of numbers of proteins per domain term. These differences are depicted in the upper graphic in the 6 pairwise Figures 1-3 (shown at the end of the Results).

Almost identically, the most abundantly found numbers of proteins (frequency ranked) unique to either SAT or VAT (Table 3) belong to the GO terms "membrane", "cytoplasm", "cytosol", "nucleus" in the domain CC, "metabolic process", "regulation of biological process", "response to stimulus", "cell organization and biogenesis" in the domain BP, and "protein binding", "catalytic activity", "nucleotide binding", "metal ion binding"( only for SAT), "RNA binding" in the domain MF (arbitrarily chosen abundant frequencies $>10 \%$; Table 3).

Differences between these most abundant frequencies of annotated numbers of proteins unique to either SAT or VAT range 0.8-10.2 PPts (percentage points; signed) and, by definition concern proteins very specific to the type of adipose tissue. These differences are depicted in the lower graphic in the 6 pairwise Figures 1-3 (shown near the end of the Results).

Interestingly, less abundant frequencies for numbers of proteins (frequency ranked) unique to either SAT or VAT (Table 3) belonging to the GO terms "extracellular" in the domain CC, "cellular component movement" in the domain BP, and "metal ion binding", "DNA binding" in the domain MF show rather large differences between frequencies.

Arbitrarily chosen large differences (i.e. > 3 PPts (signed); VAT compared with SAT) in frequencies of annotated numbers of proteins unique to either SAT or VAT were found in the GO terms "extracellular" -5.8 , "nucleus" +4.9, "cytoplasm" +3.4 , "membrane" -3.2 in the domain CC, "metabolic process" +3.0 in the domain BP, and "metal ion binding" -10.2 , "RNA binding" +7.4 , "nucleotide binding" +5.0, "protein binding"-4.5, "DNA binding" +3.5 in the domain MF (Figures 1$3)$.

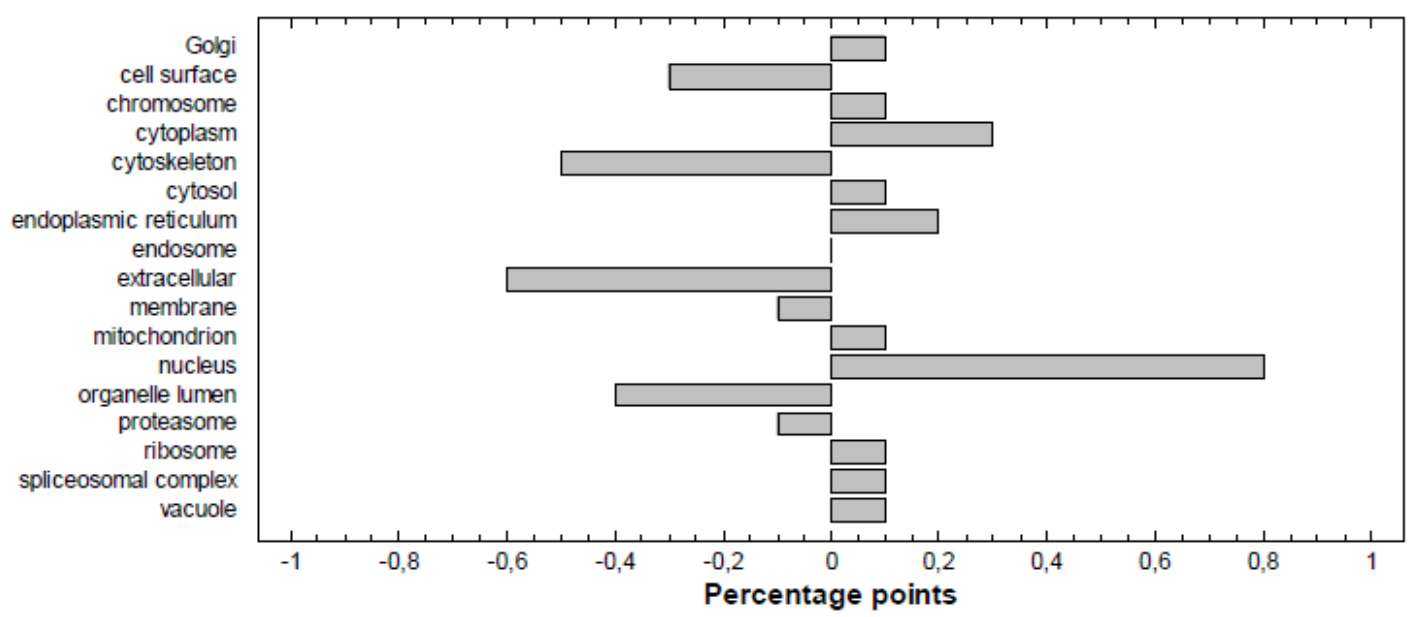

Fig1a. Difference between VAT-SAT frequency percentages of all proteins in GO domain cellular components

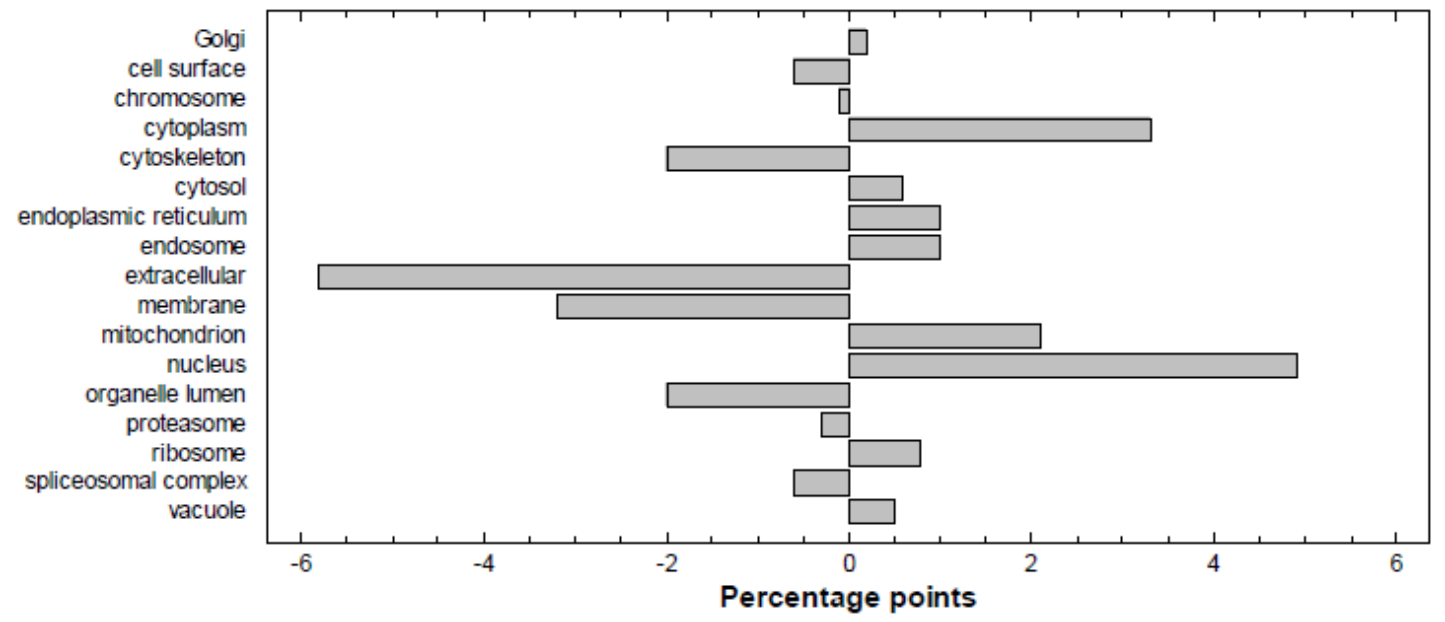

Fig1b. Difference between VAT-SAT frequency percentages of unique proteins in GO domain Cellular Components 
Paul MM van Haard et al.

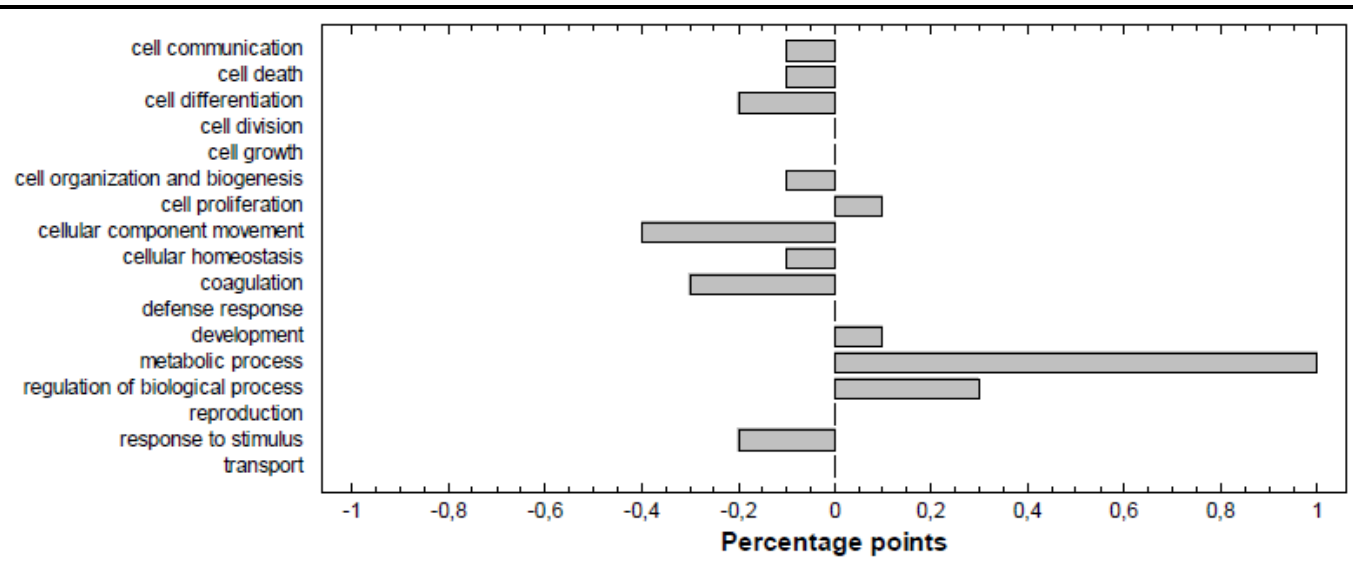

Fig2a. Difference between VAT-SAT frequency percentages of all proteins in GO domain Biological processes

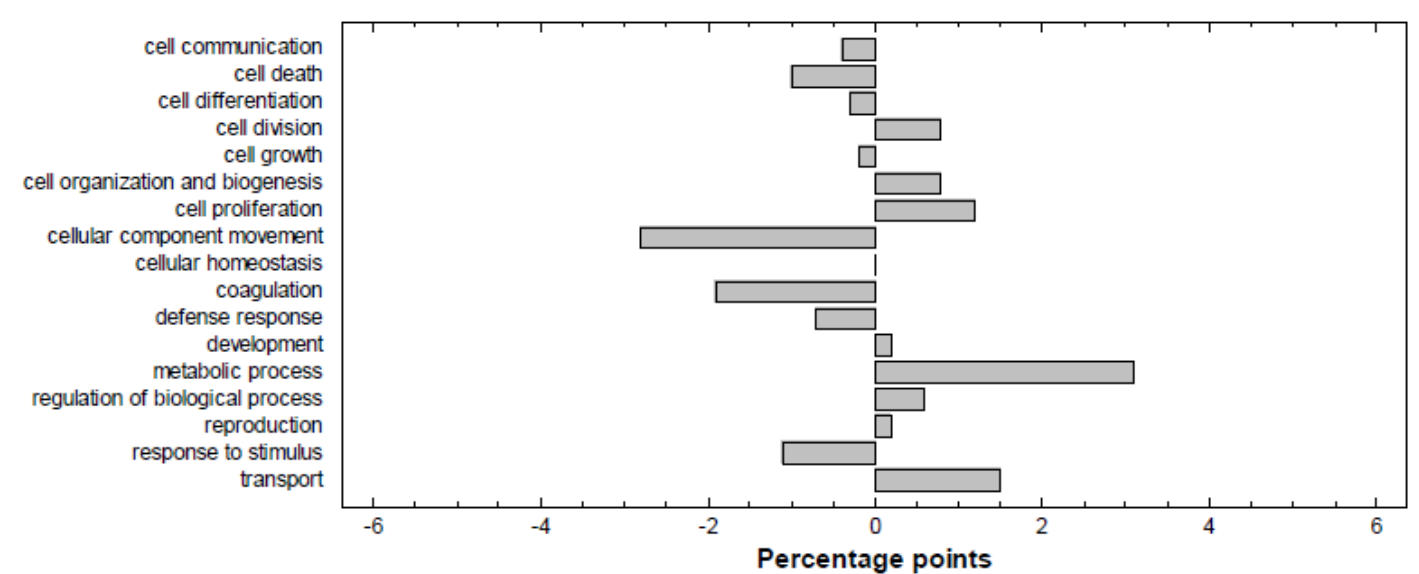

Fig2b. Difference between VAT-SAT frequency percentages of all unique proteins in GO domain Biological processes

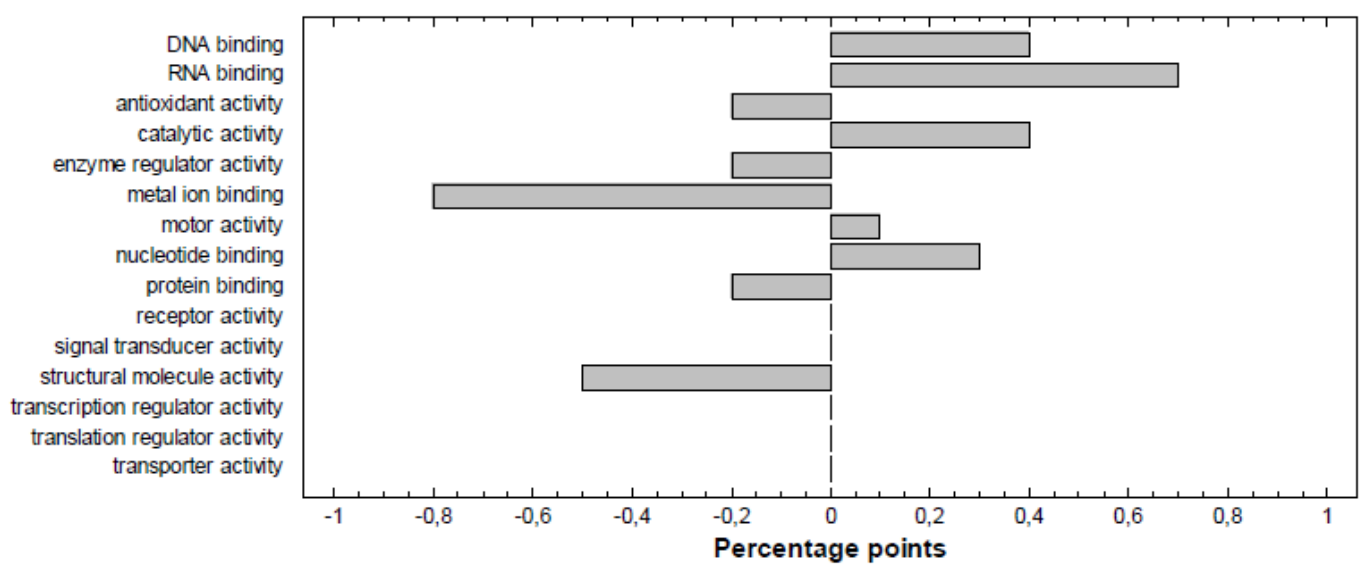

Fig3a. Difference between VAT-SAT frequency percentages of all proteins in GO domain Molecular Functions

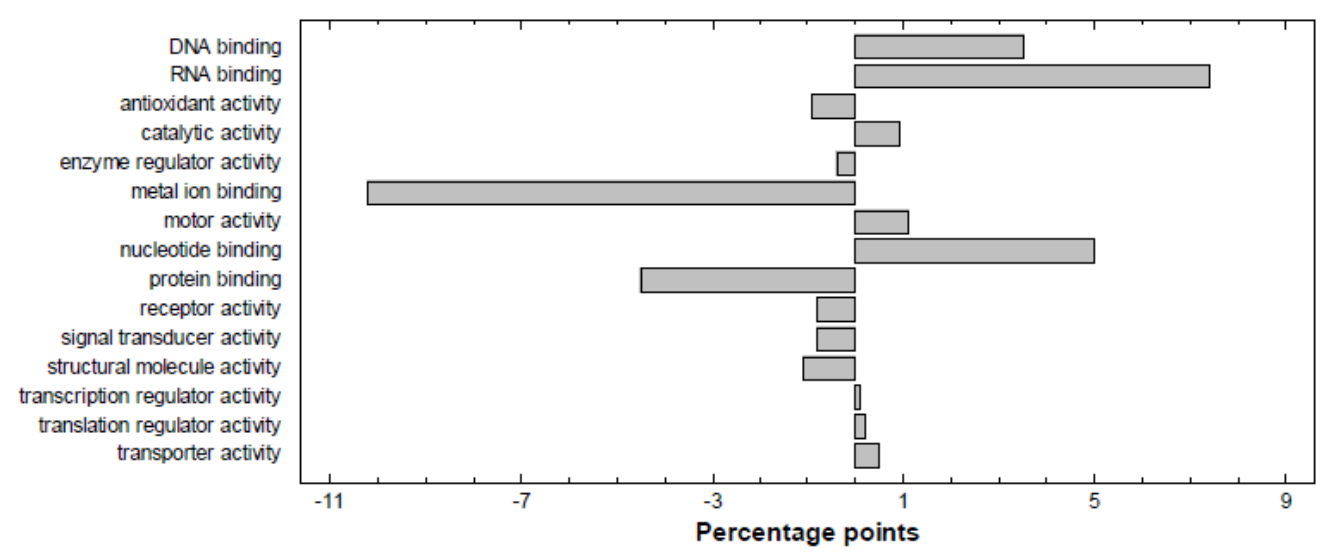

Fig3b. Difference between VAT-SAT frequency percentages of unique proteins in GO domain Molecular Function 
By definition, these differences concern differing proteins, very specific to the type of adipose tissue. Proteins (ranked firstly by GO terms, then by protein name) unique to either SAT or VAT and also those involved in substantial differences between the two tissues were identified by LC-MS/MS and can be found in the Supporting Information.

The numbers of identified proteins found were higher in VAT compared to SAT and so were also the numbers of annotated proteins. Also noted is a percentage of "unannotated" proteins (0.7-0.8\%; not shown) belonging to unknown domain terms, suggesting a need for improved protein annotation in GO domains as a whole. These "unannotated" proteins were not analyzed further.

\section{DISCUSSION}

Before starting a study on the proteome of adipose tissue one may like to estimate the maximum number of proteins to be found. The base of all potential proteins is the transcriptome: DNA is copied into molecules of ribonucleic acid (RNA), referred to as transcripts. The transcriptome is the collection of all transcripts present in a given cell or tissue. Analysis of adipose tissue gene expression data (16-20) may define indirectly the human adipose tissue proteome as studied by us. Transcriptome analysis showed that $66 \%$ of all human proteins $(n=20,344)$ may be expressed in adipose tissue (see http://www.proteinatlas.org/humanproteome/adipose ). The proteome of types of adipose tissues (this pilot study) may consist of several 100sdifferent protein species and is, therefore rather complex.

Kheterpal et al. (7) profiled the proteomes both of stromal vascular cells and mature adipocytes from human subcutaneous adipose tissue, using a quantitative 2D gel LC-MS/MS method. A total of 200 proteins were found to be differentially expressed between adipocytes and stromal vascular cells. Four adipose deposits (two types of adipose tissues) have been studied in goats recently (8) applying a holistic study approach. Ye et al. (21) quantitated nearly 1,000 protein species and found 106 significantly altered proteins during adipocyte differentiation.

The proteomics of SAT and VAT from an obese, male patient were elucidated by us with and without prior offline fractionation of proteolytic peptides, giving a representative overview of the proteomes of two paired types of human adipose tissues. The number of identified proteins found in SAT and VAT were higher in VAT compared to SAT and so were also the number of annotated proteins. Functional implications of these discrepancies are certainly interesting. A next step will be to study gender differences between SAT and VAT proteomes and the effect of diseases like type 2 diabetes mellitus, cardiovascular disease and medical interventions, i.e .caloric restriction or pharmacotherapeutic interventions (22-24). The outcome can then be used to generate a set of protein candidates for focused functional studies using the highly descriptive (GO domains terms), and semiquantitative technique (IBAQ Intensities) used in this pilot study. Based on Gene Ontology domains Cellular Components, Biological Processes, and Molecular Functions it was shown to which domains the majority of proteins are related. Our data may reflect herewith the basic relationship between and origin of SAT and VAT.

In essence, our pilot study showed for the first time major and rather large differences in frequencies of annotated numbers of proteins unique to either SAT or VAT.

\section{CONCluSion}

In the current study we were able to show for the first time common proteins as well as unique proteins annotated to the three GO domains and the domains terms, and both qualitative and semiquantitative abundances of these proteins in paired human subcutaneous and visceral white adipose tissues. The highest standard technique that we used was feasible but elaborate and expensive. It shows new aspects of adiposity research to be used in clinical protocols. More research is needed to define proteomic markers in favour of health and disease.

\section{COMPETING INTERESTS}

The authors declare no conflicts of interest. 


\section{REFERENCES}

[1] Suganami T, Tanaka M and Ogawa Y. Review: Adipose tissue inflammation and ectopic lipid accumulation. Endocrine Journal. 2012; 59(10):849-57.

[2] Hajer G, van Haeften TW, Visseren FLJ. Adipose tissue dysfunction in obesity, diabetes and vascular diseases. European Heart Journal. 2008; 29:2959-71.

[3] Suganami T, Ogawa Y. Review: Adipose tissue macrophages: their role in adipose tissue remodeling. Journal of Leukocyte Biology. 2010; 88:33-9.

[4] Chen X , Hess S. Adipose proteome analysis: focus on mediators of insulin resistance. Expert Rev Proteomics 2008; 5(6):827-39.

[5] Brockman DA, Chen X. Proteomics in the characterization of adipose dysfunction in obesity. Adipocyte. 2012;1(1):25-37.

[6] Megger DA, Bracht T, Meyer HE, Sitek B. Label-free quantification in clinical proteomics. BiochimBiophysActa. 2013; 1834(8):1581-90.

[7] Kheterpal I, Ku G, Coleman L, Yu G, Ptitsyn AA, Floyd ZE, Gimble JM. Proteome of Human Subcutaneous Adipose Tissue Stromal Vascular Fraction Cells versus Mature Adipocytes Based on DIGE. J Proteome Res. 2011; 10(4):1519-27.

[8] Restelli L, Codrea MC, Savoini G, Ceciliani F, Bendixen E. LC-MS/MS analysis of visceral and subcutaneous adipose tissue proteomes in young goats with focus on innate immunity and inflammation related proteins.J Proteomics. 2014; 108:295-305.

[9] Hess S, Chen X. In: Adipose Tissue protocols, second edition (Yang K, ed.). Applications of proteomics to the study of adipose tissue. Methods Mol Biol. 2008;456:131-40, Human Press, Totowa, NJ, USA.

[10] Wessels HJ, Gloerich J, van der Biezen E, Jetten MS, Kartal B. Liquid chromatography-mass spectrometry-based proteomics of Nitrosomonas. Methods Enzymol. 2011; 486:465-82.

[11] Rappsilber J, Ishihama Y, Mann M. Stop and go extraction tips for matrix-assisted laser desorption/ionization, nanoelectrospray, and LC/MS sample pretreatment in proteomics. Anal Chem. 2003; 75:663-70.

[12] Cox J, Mann M. MaxQuant enables high peptide identification rates, individualized ppb-range mass accuracies and proteome-wide protein quantification. Nat Biotechnol. 2008; 26:1367-72.

[13] Côté R, Reisinger F, Martens L, Barsnes H, Vizcaino JA, Hermjakob H. The Ontology Lookup Service: bigger and better.Nucleic Acids Res. 2010; May 11. PMID: 20460452.

[14] FASTA: Pearson WR,Lipman DJ. Improved Tools for Biological Sequence Analysis. PNAS 1988; 85:2444-48.

[15] Perdomo D, Aït-Ammar N, Syan S, Sachse M, Jhingan GD, Guillén N. Data set for the proteomics analysis of the endomembrane system from the unicellular Entamoeba histolytica. Data in Brief 1. 2014; 29-36; (http://dx.doi.org/10.1016/j.jprot.2014.07.034).

[16] Uhlén M, Fagerberg L, Hallström BM, et al..Proteomics. Tissue-based map of the human proteome.Science. 2015; 347(6220):1260419; doi: 10.1126/science.1260419.

[17] Perez-Perez R, Ortega-Delgado FJ et al. Differential proteomics of omental and subcutaneous adipose tissue reflects their unalike biochemical and metabolic properties. J Proteome Res. 2009;8(4): 1682-93.

[18] Peinado JR, Pardo M, de la Rosa O, Malagón MM. Proteomic characterization of adipose tissue constituents, a necessary step for understanding adipose tissue complexity. Proteomics. 2012; 12(4-5):607-20.

[19] Renes J, Mariman E. Review. Application of proteomics technology in adipocyte biology. Mol. BioSyst. 2013; 9:1076-91.

[20] Wilhelm M,Schlegl J, Hahne H,Gholami AM, Lieberenz M, Savitski MM, Ziegler E, Butzmann L, Gessulat S, Marx H,Mathieson T, Lemeer S,Schnatbaum K, Reimer U,Wenschuh H, Mollenhauer M,Slotta-Huspenina J, Boese J-H, Bantscheff M, Gerstmair A,Faerber F, Kuster B.Mass-spectrometry-based draft of the human proteome. Nature. 2014;509,582-7; doi:10.1038/ nature13319. 
[21] Ye F, Zhang H, Yang YX, et al. Comparative proteome analysis of 3T3-L1 adipocyte differentiation using iTRAQ-coupled 2D LC-MS/MS.J Cell Biochem. 2011; 112(10):3002-14.

[22] Cusi K. The Role of Adipose Tissue and Lipotoxicity in the Pathogenesis of Type 2 Diabetes. CurrDiab Rep. 2010; 10:306-15; doi: 10.1007/s11892-010-0122-6.

[23] Fain JN. Correlative studies on the effects of obesity, diabetes and hypertension on gene expression in omental adipose tissue of obese women.Nutrition and Diabetes. 2011; 1, e17; doi:10.1038/nutd.2011.14.

[24] Kelder T, Summer G, Caspers M, van Schothorst EM, Keijer J, Duivenvoorde L, Klaus S, Voigt A, Bohnert L, Pico C, Palou A, Bonet ML, Dembinska-Kiec A, Malczewska-Malec M, KiećWilk B, Del Bas JM, Caimari A, Arola L, van Erk M, van Ommen B, Radonjic M. White adipose tissue reference network: a knowledge resource for exploring health-relevant relations. Genes Nutr. 2015; 10:439; doi: 10.1007/s12263-014-0439-x. 